\title{
Reproductive outcome of a patient with congenital adrenal hyperplasia: case report
}

\author{
Bhagyashree A.*, Shylaja A. S., Yashaswini Srikonda
}

Department of Obstetrics and Gynecology, St. Philomena’s Hospital, Bangalore, Karnataka, India

Received: 20 July 2018

Accepted: 28 August 2018

\section{*Correspondence:}

Dr. Bhagyashree A,

E-mail: bhagyashree704@gmail.com

Copyright: () the author(s), publisher and licensee Medip Academy. This is an open-access article distributed under the terms of the Creative Commons Attribution Non-Commercial License, which permits unrestricted non-commercial use, distribution, and reproduction in any medium, provided the original work is properly cited.

\begin{abstract}
Congenital Adrenal Hyperplasia are a group of inherited autosomal recessive enzymatic defects of adrenal steroid biosynthesis. Low pregnancy rate has been reported in women with CAH. Several factors have been suggested to contribute to this subfertility such as androgen excess, adrenal progesterone hyper secretion, consequences of genital reconstructive surgery, secondary PCOS and psychosexual factors. In contrast to this subfertility, pregnancies are normal and uneventful. During pregnancy, maternal \& fetal problems are confined to these deficiencies. Adequate glucocorticoid therapy and improvement of surgical and psychological management could contribute to optimize fertility in $\mathrm{CAH}$. The interposition of the placenta on the HPA axis \& other endocrine changes during pregnancy impact considerably on the clinical evaluation of CAH. A 21 years old Primigravida presented to us at 13 weeks of gestation with history of $\mathrm{CAH}$ on treatment and vaginoplasty done in the childhood. Endocrinologist opinion was taken, and steroids continued. Advised to meet genetist and follow up for prenatal testing. But patient denied. Antenatal period was uneventful. Patient underwent Emergency LSCS in view of CPD in labour at 36+6 weeks of gestation. Pre-op stress dose of steroid given. A girl baby of birth weight $2.13 \mathrm{~kg}$ was born with good Apgar scores. Perioperative period uneventful. Preconception health, including hormonal, psychosexual and anatomical barriers to fertility should be addressed early in women with $\mathrm{CAH}$. This review provides fertility outcome and pregnancy issues of a woman with $\mathrm{CAH}$.
\end{abstract}

Keywords: Congenital adrenal hyperplasia, Fertility, Pregnancy

\section{INTRODUCTION}

Congenital adrenal hyperplasia $(\mathrm{CAH})$ is an autosomal recessive disorder caused by 21-hydroxylase deficiency in $90-95 \%$ cases characterized by impaired or total loss of activity in the enzyme resulting from mutations or deletions of CYP21A, required for the synthesis of cortisol and in $75 \%$ of cases, also a failure to synthesize aldosterone, which is the most severe form. ${ }^{1,2}$ Due to cortisol deficiency there is loss of negative feedback inhibition, leads to increased hypothalamic-pituitaryadrenal axis activity and subsequent hyperplasia of the adrenal gland. Clinically, 21-OHD displays heterogeneous features ranging from a classic form caused by the total absence of enzymatic function and consisting of salt wasting (SW) or the less common simple virilizing (SV) type, which has excess androgens, leading to ambiguous genitalia in newborn females and progressive postnatal virilization in males and females. ${ }^{3}$

SW form also impairs aldosterone biosynthesis resulting in severe renal salt loss and shock, unless treated during the neonatal period. The SV form has normal aldosterone levels and thus salt wasting is not present. The nonclassical form is asymptomatic or characterized by 
different hyperandrogenic signs that may manifest from childhood to adulthood.

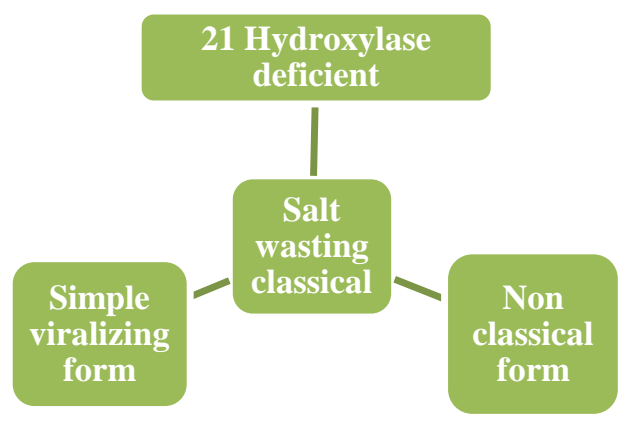

Figure 1: Congenital adrenal hyperplasia

Low pregnancy rate has been reported in women with Congenital Adrenal Hyperplasia. During pregnancy, maternal and fetal problems are confined to these deficiencies. The interposition of the placenta on the hypothalamic-pituitary- adrenal axis and other endocrine changes during pregnancy impact considerably on the clinical evaluation of CAH.

\section{CASE REPORT}

A 25years old primigravida presented to our hospital at 13 weeks of gestation for ante natal care. At 6 years of age, patient was brought by her mother to a pediatrician with complaints of ambiguous genitalia. On general physical examination clitoromegaly was noted and $\mathrm{CAH}$ was suspected.

Investigations were done showing normal FSH, normal LH, increased Testosterone, increased 17 Hydroxy progesterone and decreased serum cortisol. Scan - uterus AV, $3.7 \mathrm{~cm}$ length, bilateral ovaries normal. No testicular tissue or androgen secreting tumors were noted. Hence, diagnosed as Congenital adrenal hyperplasia.

Patient underwent clitoral resection and vaginoplasty. Patient was advised steroids supplementation, but patient stopped treatment after 2-3 months. Patient had intermittent episodes of vomiting and dehydration for which she was treated symptomatically. No history of symptoms suggestive of salt wasting were noted.

At 13 years of age, patient approached a pediatrician in view of not attaining menarche. On investigating decreased cortisol, raised DHEAS, 17 hydroxy progesterone and free testosterone were noted. Hence, started on Tab Hydrocortisone 25mg - 0 - 25mg. Patient attained menarche at 14 years of age, following which patient had regular menstrual cycles. Patient continued steroid and dose was monitored with 17 hydroxy progesterone levels and tapered accordingly. Patient was asymptomatic during treatment.

Patient had III 0 CM marriage at 20 years of age. She conceived spontaneously after one year of marital life without any assisted reproductive techniques. Patient continued to take Tab Hydrocortisone $20 \mathrm{mg}-0-10 \mathrm{mg}$ and had her first ANC visit at 13 weeks of gestation. Regular ANC investigations and NT scan were normal. As she is a known case of $\mathrm{CAH}$, Endocrinologist opinion was taken and was advised to Start Tab Prednisolone 0-0$5 \mathrm{mg} \&$ Tab Hydrocortisone 10mg (8 am)- 0-5mg (4pm).

Patient was advised to consult genesist for prenatal testing at 13 weeks of gestation, but the patient was lost to follow up and reviewed at 20 weeks of gestation. Anomaly scan showed ambiguous genitalia. As it was already too late to do prenatal testing, with reports being available after 6-8 weeks; post-natal follow up of baby was advised.

GTT at 24 weeks of gestation showed DGGT. Patient had good glycemic control on Medical Nutrional Therapy. With regular ANCs and normal interval growth scan, Patient presented to emergency with PROM at 37 weeks of gestation. Emergency cesarean section was done in view of CPD and previous vaginoplasty.

Stress dose of steroid- 100mg of IV hydrocortisone was given 1 hour prior to LSCS. She delivered a live female baby of birth weight - $2.13 \mathrm{kgs}$ with good APGAR scores. Vitals were maintained well intra operatively.

Post operatively patient was given Inj hydrocortisone $50 \mathrm{mg}$ IV 1-1-1 continued for 48 hours. Steroid dose was tapered to Tab prednisolone 5mg 0-0-1, Tab hydrocortisone $20 \mathrm{mg}-10 \mathrm{mg}-0$ and continued till postoperative day 4.

On general examination of the baby, normal genitalia were noted and was evaluated with 17 hydroxy Progesterone levels which was normal. Patient was discharged on Tab hydrocortisone $10 \mathrm{mg}-5 \mathrm{mg}-0$ and Tab prednisolone $5 \mathrm{mg} \quad 0-0-1$ with an advice of preconceptionally counseling for further pregnancies.

\section{DISCUSSION}

The incidence of classical CAH is 1:10,000-1:20,000, thus the incidence of carriers in the general population is $1: 50-1: 71$ with a median of $1: 60 .^{1,4}$

The clinical presentation of the various forms of $\mathrm{CAH}$ depends on the affected enzyme, the residual enzymatic activity, consequences of deficiencies of the end products, and hormonal effects of the elevated precursors. Deficiency of 21-hydroxylase, resulting from mutations or deletions of CYP21A, is the most common form of $\mathrm{CAH}$, accounting for more than $90 \%$ of cases. ${ }^{2}$ Due to the genetic deficiency required for biosynthesis of cortisol, 
the synthesis of cortisol was decreased, and the secretion of ACTH increased.

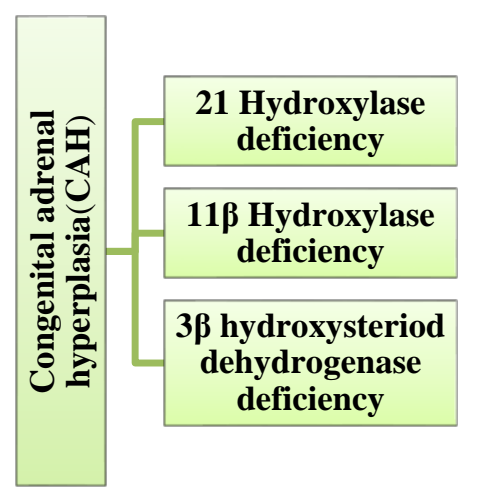

Figure 2: Types of congenital adrenal hyperplasia.

It induces the hyperplasia of adrenal cortex, resulting in characteristic enlargement of the gland. The clinical symptoms of CAH due to deficiency of 21-hydroxylase, demonstrate a wide spectrum of severity, with the most severe, classical cases diagnosed in the neonatal period due to salt-wasting and prenatal virilization in affected females, including persistence of a urogenital sinus, labioscrotal fusion and clitoromegaly. Endocrine test of 21-hydroxylase deficiency suggests that the high concentration of progesterone and 17-hydroxy progesterone in blood is significant to decide the effect of treatment because progesterone blocks the conversion to the 11-deoxycortisol. The excretion of pregnanetriol and the level of androstenedione and testosterone in urine are elevated caused by the increase of 17-hydroxy progesterone.

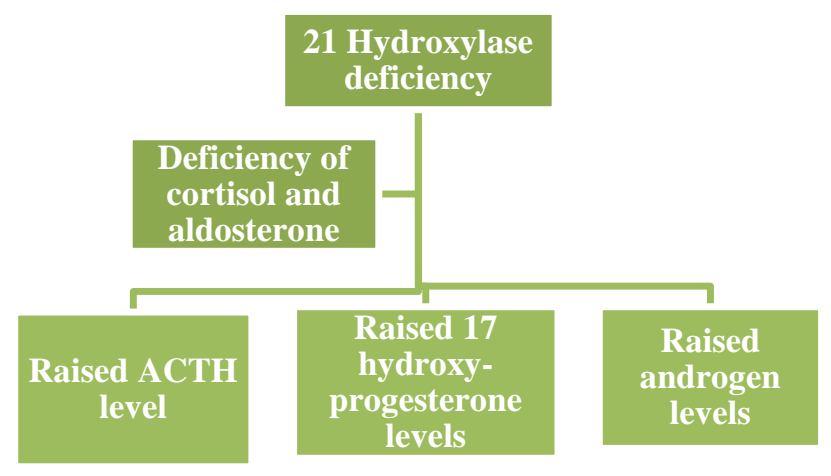

Figure 3: Changes in 21 hydroxylase deficiency.
Early diagnosis and the treatment are important which gets rid of the excessive secretion of adrenocorticotropic hormone $(\mathrm{ACTH})$, replenishes required hormone, and corrects external genitalia. Long-lasting glucocorticoid replacement treatment is the mainstay of therapy for $\mathrm{CAH}$ patients. It reduces 17-hydroxyprogesterone and adrenal androgen by normal range as glucocorticoids replacement replenishes cortisol and inhibits the secretion of ACTH.

$\mathrm{CAH}$ patients has lower pregnancy rate compared to normal women, however, cortisol replacement therapy enables the normal pregnancy, and moreover glucocorticoid treatment raises the pregnancy rate of nonclassical $\mathrm{CAH}$ patients as much as the rate of normal women.

When the fetus is at risk for $\mathrm{CAH}$, dexamethasone (20 $\mu \mathrm{g} / \mathrm{kg} /$ day in 3 divided doses) is administered to the pregnant women before the ninth week of gestation, or ideally before the seventh week, blind to the sex or affected state of the fetus. This inhibits fetal hypothalamic-pituitary-adrenal axis, excess adrenal androgen secretion and prevents virilization in affected females. Dexamethasone is used because it crosses the placenta, crossing from the maternal to the fetal circulation.

Diagnosis of CAH can be done prenatally by villous sampling or amniocentesis for mutational study or by amniotic fluid levels of 17-OHP. 1,2,5

Prenatal diagnosis is especially important if both parents are carriers; previous affected child has limited role if the mother herself is affected and in steroid treatment. Surgery of external genitalia is required to restore a female anatomy in these girls, whereas ovaries are functional and mullerian-derived internal genital structures are normal. Thus, with proper management, fertility is possible in these patients.

Diagnosis at birth is done by 17-OHP levels at day 3 of birth. In children and adults, the diagnosis is based on hormonal levels of 17-OHP, testosterone, DHEAS, androstenedione, cortisol, and plasma rennin activity. ${ }^{1,2,5}$

Treatment of classic virilizing $\mathrm{CAH}$ consists of glucocorticoids and genitoplasty. ${ }^{4,6}$ Fertility is reduced in females of $\mathrm{CAH}$ because of various reasons like psychosexual problems, inadequate vaginal introitus, coital difficulties, chronic oligo-anovulation, secondary PCOS and luteal phase defect. ${ }^{4}$

Fertility rates of $60-80 \%$ and $7-60 \%$ have been reported in women with classic SV and classic SW CAH, respectively. ${ }^{5}$

In contrast to this sub-fertility, pregnancies are commonly normal and uneventful. Thus, fertility rather than 
pregnancy rates seem to be reduced in women with classical CAH compared to the general population.

Studies have shown that proper treatment results in spontaneous conception in patients with $\mathrm{CAH}$. Adult patients with $\mathrm{CAH}$ have a number of issues due either to the disease or to its treatment. Most of the problems relate to final height, fertility, bone metabolism and psychoneurological issues. During labor and delivery, the mother should receive increased doses of hydrocortisone. Women with $\mathrm{CAH}$ often have android pelvis characteristics, increasing the risk for cephalo-pelvic disproportion and dystocia. ${ }^{7,8}$ Elective caesarean section should be considered especially for those who have had reconstructive surgery of external genitalia.

Although there have been few reports of masculinization of external genitalia, this problem seems to be very rare, and girls born to women with $\mathrm{CAH}$ are generally unaffected. In fact, the placenta serves as a metabolic barrier and reduces fetal exposure to maternal androgens through placental aromatization of maternal testosterone and androstenedione.

Other maternal factors that can contribute to fetal protection include a reduction in bioavailable testosterone due to increased sex hormone-binding globulin levels and the potential anti- androgenic effects of progesterone. ${ }^{7,8}$

\section{CONCLUSION}

Preconception health, including hormonal, psychosexual and anatomical barriers to fertility should be addressed early in women with classical congenital adrenal hyperplasia $(\mathrm{CAH})$. Fertility and pregnancy outcomes improve with adequate replacement of glucocorticoids and suppression of adenocorticotropic hormone (ACTH), progesterone and androgen production. Patients with $\mathrm{CAH}$ should be regularly followed up from childhood to adulthood by multidisciplinary teams. Optimal replacement therapy, close clinical and laboratory monitoring, early life-style interventions, early and regular fertility assessment and continuous psychological management are needed to improve outcome.

\section{ACKNOWLEDGMENTS}

It is a great pleasure to express sincere thanks to all those who managed this case very well. A special thanks to the endocrinologist Dr. Sudeep for managing this case very well.

Funding: No funding sources

Conflict of interest: None declared

Ethical approval: Not Required

\section{REFERENCES}

1. Al-Agha, A., A. Ocheltree, and M. Al-Tamimi, Association between genotype, clinical presentation, and severity of congenital adrenal hyperplasia: a review. Turkish J Pediat, 2012.54(4):323-32.

2. Lin-Su K, Nimkarn S, New MI. Congenital adrenal hyperplasia in adolescents: diagnosis and management. Ann N Y Acad Sci 2008;1135(1):95-8.

3. Belinda G, Vinay D, Moolechery J, Mathew V, Anantharaman R, Ayyar V, et al Congenital adrenal hyperplasia -experience from a tertiary centre in South India. IndianJournal of Endocrinology and Metabol. 2012;16(2):S385-6.

4. Mahdi Kamoun, M.M.F., Mohamed Habib Sfar, and Mohamed Abid, Congenital adrenal hyperplasia: Treatment and outcomes. Indian J Endocrinol Metabol. 2013.17(1):p.S14-17.

5. Mulaikal RM, Migeon CJ, Rock JA. Fertility rates in female patients with congenital adrenal hyperplasia due to 21-hydroxylase deficiency.N Engl J Med 1987;316:178-82.

6. Gonzalez, R. and B. Ludwikowski, Should the genitoplasty of girls with CAH be done in one or two stages? Frontiers in Pediatrics, 2014.1(54):1-2.

7. Ambroziak U, Bednarczuk T, Ginalska-Malinowska M, Małunowicz EM, Grzechocińska B, Kamiński P, et al. Congenital adrenal hyperplasia due to 21hydroxylase deficiency-management in adults. Endokrynol Pol. 2010;61(1):142-55.

8. Witchel SF. Management of CAH during pregnancy: Optimizing outcomes. Curr Opin Endocrinol Diabetes Obes. 2012;19(6):489-96.

Cite this article as: Bhagyashree A, Shylaja AS, Yashaswini S, Reproductive outcome of a patient with congenital adrenal hyperplasia: Case Report. Int J Reprod Contracept Obstet Gynecol 2018;7:4297300. 